A $\mathrm{C}$ G G Rec. Nat. Prod. 14:5 (2020) 383-386
records of natural products

\title{
Scoparic acid E: A New Labdane Diterpenoid on Attenuating Palmitate Induced Viability in MIN6 Cells from Scoparia dulcis Caiyun Zhang $\odot^{1 \#}$, Lizhen Chen $\odot^{1 \#,}$, Yiying Li $\odot^{2}$, Na Wei $\odot{ }^{1}$,
}

\author{
Lu Zhang $\oplus^{1}$, Lin Dong $\oplus^{1}$, Yong Zhang $\oplus^{* 2}$ and Xiaopo Zhang $\oplus^{1 *}$ \\ ${ }^{1}$ Key Laboratory of Tropical Translational Medicine of the Ministry of Education, Hainan Key \\ Laboratory for Research and Development of Tropical Herbs, School of Pharmacy, Hainan Medical \\ University, Haikou 571199, P.R. China \\ ${ }^{2}$ Department of Basic Medicine and Life Sciences, Hainan Medicinal University, Haikou 571199, \\ P.R.China
}

(Received February 24, 2020; Revised March 20, 2020; Accepted March 25, 2020)

\begin{abstract}
A new labdane diterpenoid, named Scoparic acid E (1), was obtained from the medicinal plant of Scoparia dulcis L. The structure of Scoparic acid E (1) was elucidated and characterized through detailed physical analyses of its NMR spectroscopic data and HRMS. Scoparic acid E (1) was tested for its effect on attenuating palmitate-induced viability at 25 and $50 \mu \mathrm{M}$. The results showed that compound 1 significantly attenuated palmitate-induced viability in MIN6 cells.
\end{abstract}

Keywords: Scoparia dulcis L.; scoparic acid E; labdane diterpenoid; palmitate-induced viability. @ 2020 ACG Publications. All rights reserved.

\section{Plant Source}

The fresh plant of Scoparia dulcis L. was obtained from Wuzhi Mountain of Hainan Island, China, in July 2018. This medicinal plant was authenticated by Dr. Yuguang Fan, and a voucher specimen has been deposited with a documented number of No.SD201807.

\section{Previous Studies}

S. dulcis, belonging to the Scrophulariaceae family, is native to Hainan Island and abound in natural resources. It is commonly used as alternatives for the treatment of many illnesses $[1,2]$. Pharmacological investigations indicated that the extract of $S$. dulcis possessed anti-diabetic, antiinflammatory, antioxidant, anti-tumour, and other properties [3,4]. The previous investigations showed that $S$. dulcis contains diterpenoids such as Scoparic acids A-D, alkaloids, flavonoids, and other chemical constituents [5-7].

\footnotetext{
${ }^{*}$ Corresponding authors: E-mail: 935150242@qq.com (Y.Zhang); E-mail: z_xp1412@163.com (X.P. Zhang)

\# Authors contributed equally to this paper.
} 


\section{Present Study}

In present study, we investigate the chemical constituents of Scoparia dulcis L. By this process, a new labdane diterpenoid denoted scoparic acid E (1) was obtained and structurally elucidated. Its biological activity on attenuating palmitate-induced viability in MIN6 cells was also reported herein.

Dried S. dulcis $(10.0 \mathrm{~kg})$ was cut into pieces and extracted by a mixture of ethanol-water $(8: 2)$ for three times to give an extract $(1200 \mathrm{~g})$. The extract was dissolved in $\mathrm{H}_{2} \mathrm{O}$ and partitioned by petroleum for three times to remove the hydrophobic constituents. The mother-liquid was then partitioned by $\mathrm{CH}_{2} \mathrm{Cl}_{2}$ for three times. The $\mathrm{CH}_{2} \mathrm{Cl}_{2}$ solution was concentrated to produce the $\mathrm{CH}_{2} \mathrm{Cl}_{2}$ extract. The $\mathrm{CH}_{2} \mathrm{Cl}_{2}$ extract was coarsely isolated and purified. A gradient elution of petroleumdichloromethane-acetone was applied to elute the chemical constitutes in the silica gel column, which gave six fractions (Fra.1-Fra.6). Fra.3 was further purified by the same step as above using petroleumdichloromethane-acetone $(5: 1: 1)$ as the eluent to afford six fractions (Subfra.1-Subfra.6). Subfra.4 $(1.0 \mathrm{~g})$ was successively separated by a column Sephadex LH-20 and finally isolated by HPLC using a mixture of methanol-water (60:40) to afford $\mathbf{1}(10.0 \mathrm{mg})$. The retention time of compound $\mathbf{1}$ is 53.5 min under the above HPLC conditions.

Scoparic acid $E(\mathbf{1})$ : White powder, $[\alpha]^{25}{ }_{D}=-12$ (c 0.05), UV (MeOH) $\lambda_{\max }(\log \varepsilon): 228$ (3.80), 276 $(2.34) \mathrm{nm} .{ }^{1} \mathrm{H}$ NMR $\left(600 \mathrm{MHz}, \mathrm{CDCl}_{3}\right) \delta(\mathrm{ppm})=1.74(1 \mathrm{H}, \mathrm{m}, \mathrm{H}-1 \alpha), 1.20(1 \mathrm{H}$, brd, $J=13.2 \mathrm{~Hz}, \mathrm{H}-$ $1 \beta), 1.78(1 \mathrm{H}, \mathrm{m}, \mathrm{H}-2 \beta), 1.61(1 \mathrm{H}, \mathrm{m}, \mathrm{H}-2 \alpha), 1.72(2 \mathrm{H}, \mathrm{m}, \mathrm{H}-3), 2.65(1 \mathrm{H}$, brs, H-5), 5.47 (1H, brs, H6), $2.60(1 \mathrm{H}$, brd, $J=13.2 \mathrm{~Hz}, \mathrm{H}-7 \alpha), 2.48(1 \mathrm{H}$, brd, $J=13.2 \mathrm{~Hz}, \mathrm{H}-7 \beta), 1.93$ (1H, brs, H-9), 1.97 (1H, m, H-11a), $1.68(1 \mathrm{H}, \mathrm{m}, \mathrm{H}-11 \mathrm{~b}), 2.22(1 \mathrm{H}, \mathrm{t}, J=13.2 \mathrm{~Hz}, \mathrm{H}-12 \mathrm{a}), 2.10(1 \mathrm{H}, \mathrm{m}, \mathrm{H}-12 \mathrm{~b}), 5.67$ (1H, s, H-16a), 6.26 (1H, s, H-16b), $4.76(2 \mathrm{H}, \mathrm{H}-17), 1.36$ (3H, s, H-19), 1.50 (3H, s, H-20), 8.04 (2H, d, $\left.J=8.4 \mathrm{~Hz}, \mathrm{H}-2^{\prime}, 6^{\prime}\right), 7.56\left(1 \mathrm{H}, \mathrm{t}, J=8.4 \mathrm{~Hz}, \mathrm{H}-4^{\prime}\right), 7.46\left(2 \mathrm{H}, \mathrm{t}, J=8.4 \mathrm{~Hz}, \mathrm{H}-3^{\prime}, 5^{\prime}\right) .{ }^{13} \mathrm{C}$ NMR $(150$ $\left.\mathrm{MHz}, \mathrm{CDCl}_{3}\right) \delta(\mathrm{ppm})=37.8(\mathrm{C}-1), 18.2(\mathrm{C}-2), 40.0(\mathrm{C}-3), 47.0(\mathrm{C}-4), 42.3$ (C-5), 74.0 (C-6), 37.0 (C-7), 144.4 (C-8), 57.4 (C-9), 38.6 (C-10), 26.8 (C-11), 32.4 (C-12), 140.7 (C-13), 172.9 (C-14), 127.6 (C-16), 113.3 (C-17), 184.7 (C-18), 19.2 (C-19), 25.6 (C-20), 129.6 (C-1'), 130.7 (C-2', 6'), 128.4 (C-3', 5'), 132.8 (C-4'), 166.2 (C-7'). HRESIMS: $m / z 439.2130$ ([M - H]', calcd. $\mathrm{C}_{26} \mathrm{H}_{31} \mathrm{O}_{6}$ for 439.2121).

Bioactivity Test-Cell Viability Assay: The testing method was applied as previous report [10]. MIN6 cells were incubated with the absence of 25 and $50 \mu \mathrm{M}$ of Scoparic acid E and then the cell viability was measured.

The dried $S$. dulcis plant was cut into small pieces and extracted under reflux. The solvent was quickly evaporated to produce an extract, which was processed by a series of isolation procedures to afford Scoparic acid E (Figure 1).

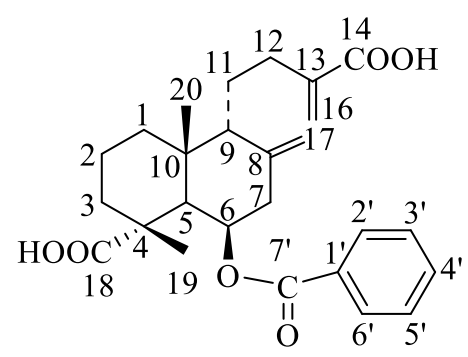

Figure 1. Structure of Scoparic acid E (1) isolated from S. dulcis

Compound 1, scoparic acid E, has a molecular formula $\mathrm{C}_{26} \mathrm{H}_{32} \mathrm{O}_{6}$ as established by HRESIMS at $\mathrm{m} / z$ 439.2130 [M - H] ${ }^{-}$(calcd. for 439.2121). The ${ }^{1} \mathrm{H}$ NMR spectral data showed resonances for two methyls $\left[\delta_{\mathrm{H}} 1.36(\mathrm{~s}, \mathrm{H}-19), 1.50(\mathrm{~s}, \mathrm{H}-20)\right]$, a set of benzoyl protons $\left[\delta_{\mathrm{H}} 8.04\left(\mathrm{~d}, J=8.4 \mathrm{~Hz}, \mathrm{H}-2^{\prime}, 6^{\prime}\right)\right.$, 
$7.56\left(\mathrm{t}, J=8.4 \mathrm{~Hz}, \mathrm{H}-4^{\prime}\right), 7.46\left(\mathrm{t}, J=8.4 \mathrm{~Hz}, \mathrm{H}-3^{\prime}, 5^{\prime}\right)$ ], two exocyclic methylenes [ $\delta_{\mathrm{H}} 4.76(\mathrm{H}-17), 5.67$ (H-16a), $6.26(\mathrm{H}-16 \mathrm{~b})]$, six methylenes [ $\delta_{\mathrm{H}} 1.74(\mathrm{~m}, \mathrm{H}-1 \alpha), 1.20$ (brd, $\left.J=13.2 \mathrm{~Hz}, \mathrm{H}-1 \beta\right), 1.78(\mathrm{~m}, \mathrm{H}-$

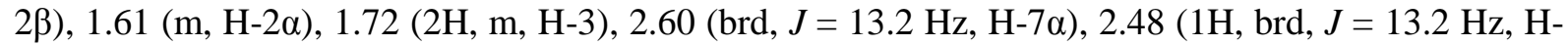
7ß), 1.97 (m, H-11a), 1.68 (m, H-11b), 2.22 (t, $J=13.2 \mathrm{~Hz}, \mathrm{H}-12 \mathrm{a}), 2.10$ (m, H-12b)], three methines [ $\delta_{\mathrm{H}} 2.65$ (brs, H-5), 5.47 (brs, H-6), 1.93 (brs, H-9)]. The ${ }^{13} \mathrm{C}$ NMR spectrum, associated with HSQC experiments, resolved 26 carbon resonances attributable to one benzoyl group [129.6 (C-1'), 130.7 (C2', 6'), $128.4\left(\mathrm{C}-3^{\prime}, 5^{\prime}\right), 132.8\left(\mathrm{C}^{\prime} 4^{\prime}\right), 166.2\left(\mathrm{C}^{\prime} 7^{\prime}\right)$ ], two methyls [ $\left.\delta_{\mathrm{C}} 19.2(\mathrm{C}-19), 25.6(\mathrm{C}-20)\right]$, six methylenes $\left[\delta_{\mathrm{C}} 37.8(\mathrm{C}-1), 18.2(\mathrm{C}-2), 40.0(\mathrm{C}-3), 37.0(\mathrm{C}-7), 26.8(\mathrm{C}-11), 32.4(\mathrm{C}-12)\right]$, three methines [ $\left.\delta_{\mathrm{C}} 42.3(\mathrm{C}-5), 74.0(\mathrm{C}-6), 57.4(\mathrm{C}-9)\right]$, two tertiary carbons $\left[\delta_{\mathrm{C}} 47.0(\mathrm{C}-4), 38.6(\mathrm{C}-10)\right]$, two carboxyl groups $\left[\delta_{\mathrm{C}} 172.9(\mathrm{C}-14), 187.4(\mathrm{C}-18)\right]$, and two double bonds $\left[\delta_{\mathrm{C}} 146.7(\mathrm{C}-8), 114.3(\mathrm{C}-17)\right.$, 140.7 (C-13), $127.6(\mathrm{C}-16)]$.

The observed ${ }^{1} \mathrm{H}-{ }^{1} \mathrm{H}$ COSY spectroscopic data indicated the three units of $\mathrm{C}_{1}-\mathrm{C}_{2}-\mathrm{C}_{3}$, and $\mathrm{C}_{5}-\mathrm{C}_{6^{-}}$ $\mathrm{C}_{7}$, and $\mathrm{C}_{9}-\mathrm{C}_{11}-\mathrm{C}_{12}$ as depicted in Figure 2. The HMBC spectrum of $\mathbf{1}$ exhibited correlations from $\delta_{\mathrm{H}}$ $1.75(\mathrm{H}-3)$ to signals at $\delta_{\mathrm{C}} 47.0(\mathrm{C}-4), \delta_{\mathrm{C}} 42.3(\mathrm{C}-5)$, and correlations from $\delta_{\mathrm{H}} 1.36(\mathrm{H}-19)$ to carbons of C-4, C-5 and C-18 ( $\left.\delta_{\mathrm{C}} 187.4\right)$ allowed the elucidation of connections from C-1 to C-5. HMBC connections of the methyl resonance at $\delta_{\mathrm{H}} 1.50(\mathrm{H}-20)$ to carbons at $\delta_{\mathrm{C}} 37.8(\mathrm{C}-1), 38.6(\mathrm{C}-10), 42.3$ $(\mathrm{C}-5)$, and correlations from proton at $\delta_{\mathrm{H}} 1.93(\mathrm{H}-9)$ to $\delta_{\mathrm{C}} 146.7(\mathrm{C}-8), 38.6(\mathrm{C}-10), 26.8(\mathrm{C}-11), 32.4$ (C-12) allowed the establishment of the carbon skeleton of 1 [8]. The HMBC connections from $\delta_{\mathrm{H}}$ $2.22,2.10(\mathrm{H}-12)$ and $\delta_{\mathrm{H}} 6.26,5.67(\mathrm{H}-16)$ to the carboxyl group of $\delta_{\mathrm{C}} 172.9(\mathrm{C}-14)$ and correlation from $\delta_{\mathrm{H}} 5.47(\mathrm{H}-6)$ to $\delta_{\mathrm{C}} 129.6(\mathrm{C}-1$ ') showed 1 have a same skeleton with Scoparic acid C, except for the aldehyde group of $\mathrm{C}-14$ which changed into the carboxyl group in 1 [9].

The relative configurations of compound $\mathbf{1}$ was diagnosed according to ROESY correlations as the similar compounds [9]. $\mathrm{H}-20\left(\delta_{\mathrm{H}} 1.50\right), \mathrm{H}-19\left(\delta_{\mathrm{H}} 1.36\right)$, and $\mathrm{H}-9\left(\delta_{\mathrm{H}} 1.93\right)$ were determined to be $\beta$ orientated, and $\mathrm{H}-5\left(\delta_{\mathrm{H}} 2.65\right), \mathrm{H}-6\left(\delta_{\mathrm{H}} 5.47\right)$ were arranged in $\alpha$-orientation. Thus, compound 1 was established with a given name Scoparic acid E as depicted in Figure 1.

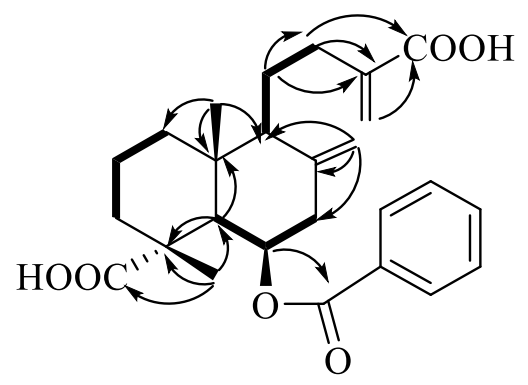

Figure 2. Key ${ }^{1} \mathrm{H}-{ }^{1} \mathrm{H}-\mathrm{COSY}$ (bold) and HMBC (arrow) connections for Scoparic acid E (1)

Scoparic acid E was tested for its effect on attenuating palmitate-induced viability in MIN6 cells. The result showed that compound $\mathbf{1}$ significantly attenuated the viability by palmitate-induced decrease in MIN6 cells as shown in Figure 3.

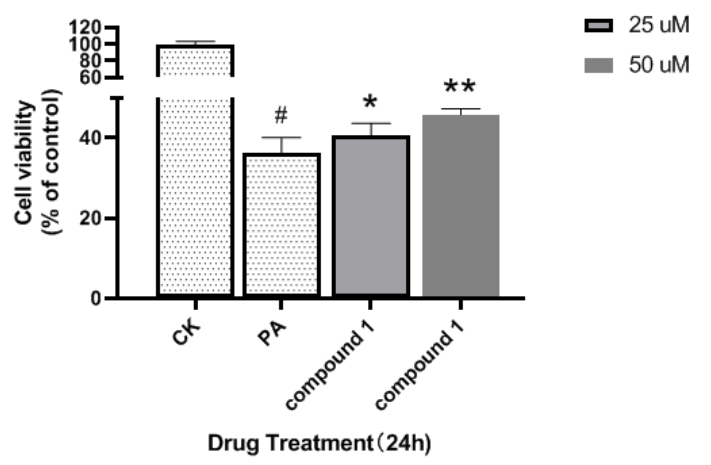

Figure 3. Cell viability treated by Scoparic acid E (1) in MIN6 cells. \# $p<0.01$ compared with control, ${ }^{*} \mathrm{p}<0.05$ compared with PA, ${ }^{*} \mathrm{p}<0.01$ compared with PA. 


\section{Acknowledgments}

This work was supported by the National Natural Science Fund for the National Science Foundation of China (No. 81760628, 81560696)

\section{Supporting Information}

Supporting Information accompanies this paper on http://www.acgpubs.org/journal/recordsof-natural-products

\section{ORCID}

Caiyun Zhang: 0000-0002-4432-5320

Lizhen Chen: 0000-0001-5335-0299

Yiying Li: $0000-0001-6855-522 \mathrm{X}$

Na Wei: 0000-0002-5543-7205

Lu Zhang:0000-0001-9072-4974

Lin Dong: 0000-0003-0877-349X

Yong Zhang: 0000-0001-5333-7684

Xiaopo Zhang: 0000-0003-2614-7425

\section{References}

[1] M. Latha, K. M. Ramkumar, L. Pari, P. N. Damodaran, V. Rajeshkannan and T. Suresh (2006). Phytochemical and antimicrobial study of an antidiabetic plant: Scoparia dulcis L., J. Med. Food 9, 391394.

[2] J. C. Tsai, W. H. Peng, T. H. Chiu, S. H. Huang, T. H. Huang, S. H. Lai, Z. R. Lai and C. Y. Lee (2010). Hepatoprotective effect of Scoparia dulcis on carbon tetrachloride induced acute liver injury in mice, Am. J. Chinese Med. 38, 761-775.

[3] M. Latha, L. Pari, K. M. Ramkumar, P. Rajaguru, T. Suresh, T. Dhanabal, S. Sitasawad and R. Bhonde (2009). Antidiabetic effects of scoparic acid D isolated from Scoparia dulcis in rats with streptozotocininduced diabetes, Nat. Prod. Res. 23, 1528-1540.

[4] W. H. Wu, Y. T. Chen, R. W. Lu, S. T. Chen and C. C. Chang (2012). Benzoxazinoids from Scoparia dulcis (sweet broomweed) with antiproliferative activity against the DU-145 human prostate cancer cell line, Phytochemistry 83, 110-115.

[5] S. Mandal, C. Kumar, A. Majumder, R. Majumder and B. Maity (2000). Antibacterial activity of Litsea glutinosa bark, Fitoterapia 71, 439-441.

[6] M. Ahsan, S. K. Islam, A. I. Gray and W. H. Stimson (2003). Cytotoxic diterpenes from Scoparia dulcis, J. Nat.Prod. 66, 958-961.

[7] Y. Li, X. Chen, M. Satake, Y. Oshima and Y. Ohizumi (2004). Acetylated flavonoid glycosides potentiating NGF action from Scoparia dulcis, J. Nat.Prod. 67, 725-727.

[8] L. Y. Kong, M. J. Qin and M. Niwa (2000). Diterpenoids from the Rhizomes of Alpinia calcarata, J. Nat.Prod. 63, 939-942.

[9] T. Hayashi, M. Kawasaki, K. Okamura, Y. Tamada, N. Morita, Y. Tezuka and T. Kikuchi (1992). Scoparic acid A, a $\beta$-glucuronidase inhibitor from Scoparia dulcis, J. Nat.Prod. 55, 1748-1755.

[10] F. Y. Guan, J. Gu, W. Li, M. Zhang, Y. S. Ji, J. Li, L. Chen and G. M. Hatch (2014). Compound K protects pancreatic islet cells against apoptosis through inhibition of the AMPK/JNK pathway in type 2 diabetic mice and in MIN6 $\beta$-cells, Life Sci. 107, 42-49.

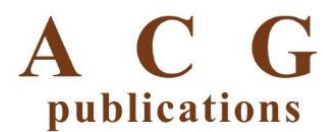

(C) 2020 ACG Publications 